\title{
Duodenum Exclusion Alone Is Sufficient to Reduce Fasting Blood Glucose in Non-Obese Diabetic Goto-Kakizaki Rats
}

\author{
Jun Ke • Yu Wang
}

Published online: 5 January 2014

(C) The Author(s) 2014. This article is published with open access at Springerlink.com

To the editor,

In addition to reducing weight, bariatric surgery could improve glucose metabolism in patients with type 2 diabetes mellitus $[1,2]$. Such actions have mainly been explained by the foregut and the hindgut hypotheses. The foregut hypothesis claims that bariatric surgery removes endogenous substances that cause disturbance in glucose metabolism [3]. The hindgut hypothesis postulates that the glucose-lowering action is mainly the result of the expedited food delivery to the hindgut [4]. However, effects of gastric bypass surgery on glucose metabolism and the relative contribution of the foregut vs. hindgut hypothesis vary considerably based on the type of the surgery. Another major confounding factor is the reflux of gastrointestinal (GI) content into the duodenum $[5,6]$.

In this preliminary rat study, we examined whether duodenum exclusion alone is sufficient to improve glucose metabolism in a rat model of diabetes. Briefly, the intestine of nonobese diabetic Goto-Kakizaki (GK) rats was transected immediately below the ligament of Treitz. A 3-mm silicon tube was used to connect the duodenum with jejunum and fixed in the wall of jejunum (Fig. 1a). Short-term mortality $(<1$ week) in this pilot experiment was $100 \%(n=55$; Table 1$)$. We therefore modified the procedure by wrapping a shorter silicon tube in the duodenum and ligation of the distal end of the duodenum ( $n=20$; Fig. 1b) and achieved a $90 \%$ survival (18/20). Such a

J. Ke

Department of General Surgery, Fuzhou General Hospital, Fujian University of Traditional Chinese Medicine, Fuzhou, Fujian 350025, China

Y. Wang $(\bowtie)$

Department of General Surgery, Fuzhou General Hospital of Nanjing Command, Fuzhou, Fujian 350025, China

e-mail: flyfishwang@hotmail.com modified surgery resulted in a significant reduction of fasting plasma glucose in the following weeks (Fig. 2).
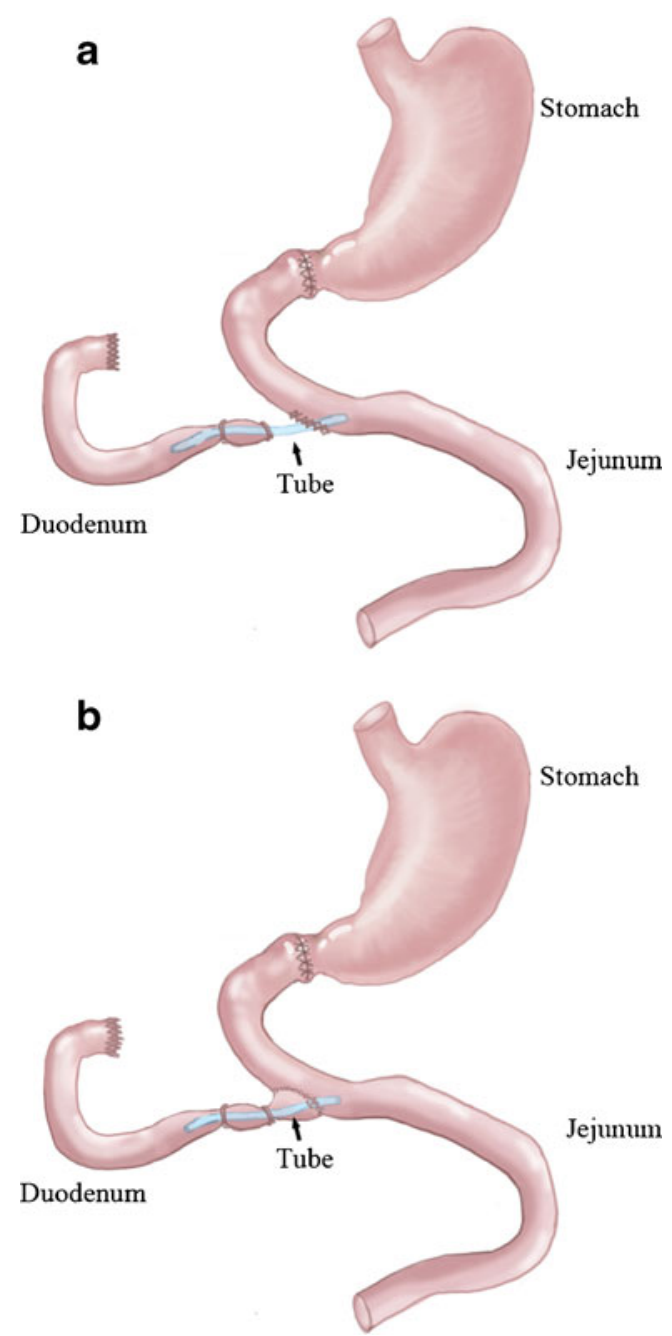

Fig. 1 A schematic illustration of duodenum exclusion (a) and modified duodenum exclusion (b) 
Table 1 Cause of death

\begin{tabular}{lcl}
\hline Type & $\begin{array}{l}\text { Duodenum } \\
\text { exclusion }\end{array}$ & $\begin{array}{l}\text { Modified duodenum } \\
\text { exclusion }\end{array}$ \\
\hline Anesthesia & 1 & 0 \\
Bleeding & 4 & 0 \\
Fistula & 5 & 0 \\
Adhesion & 41 & 0 \\
Intestinal obstruction & 1 & 2 \\
Unknown & 3 & 0 \\
Total & 55 & 2 \\
\hline
\end{tabular}

In this study, the surgical intervention completely excluded the duodenum and prevented the possibility of reflux. The results showed that duodenum exclusion alone is sufficient to reduce blood glucose. To further examine the validity of the model, we are currently conducting a series of experiments to expand the finding to other relevant measures (such as insulin

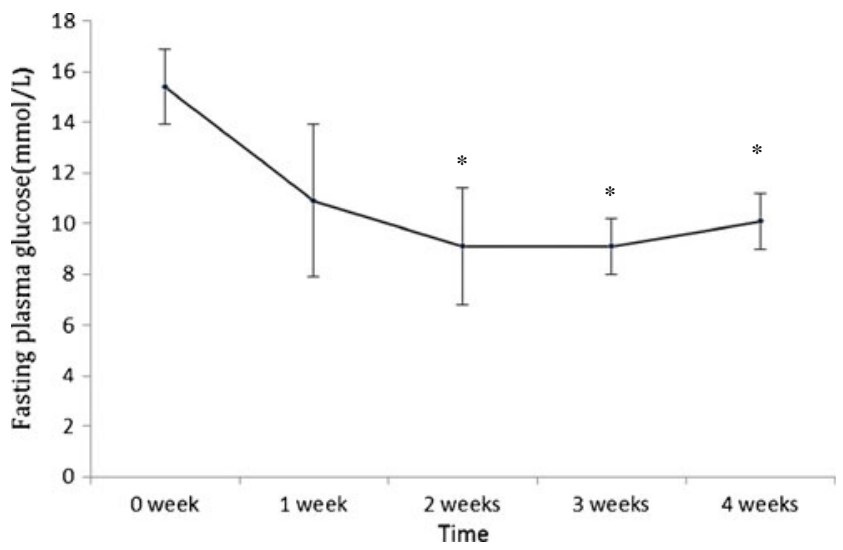

Fig. 2 Data are expressed as mean $\pm \mathrm{SD}$ and analyzed with pairwise $t$ test. $* p<0.05$ vs. presurgery baseline tolerance) and to examine the effects of establishing the normal GI integrity in rats with duodenum exclusion.

In summary, this surgery is challenging but feasible for experienced surgeons and may provide an alternative animal model to study the action of bariatric surgery.

Acknowledgments This study was supported by the Fujian Provincial Science Cooperation Project (201210007), the Fujian Provincial Key Science and Technology Project (2009Y0039) and the Nanjing Command Key Medical Project (09Z031).

Conflict of Interest None of the authors had conflicts of interest.

Open AccessThis article is distributed under the terms of the Creative Commons Attribution License which permits any use, distribution, and reproduction in any medium, provided the original author(s) and the source are credited.

\section{References}

1. Buchwald H, Avidor Y, Braunwald E, et al. Bariatric surgery: a systematic review and meta-analysis. JAMA. 2004;292(14): 1724-37. PMID: 15479938.

2. Zhu L, Mo Z, Yang X, et al. Effect of laparoscopic Roux-en-Y gastroenterostomy with $\mathrm{BMI}<35 \mathrm{~kg} / \mathrm{m}(2)$ in type 2 diabetes mellitus. Obes Surg. 2012;22(10):1562-7. PMID: 22692669.

3. Rubino F, Forgione A, Cummings DE, et al. The mechanism of diabetes control after gastrointestinal bypass surgery reveals a role of the proximal small intestine in the pathophysiology of type 2 diabetes. Ann Surg. 2006;244(5):741-9. PMID: 17060767.

4. Patriti A, Aisa MC, Annetti C, et al. How the hindgut can cure type 2 diabetes. Ileal transposition improves glucose metabolism and betacell function in Goto-Kakizaki rats through an enhanced proglucagon gene expression and L-cell number. Surgery. 2007;142(1):74-85. PMID: 17630003.

5. Moffat RE, Peltier GL, Jewell WR. The radiological spectrum of gastric bypass complications. Radiology. 1979;132(1):33-6. PMID: 451218.

6. Fobi MA, Lee H, Holness R, et al. Gastric bypass operation for obesity. World J Surg. 1998;22(9):925-35. 